\title{
APLICAÇÃO DE GLICERINA RESIDUAL DA PRODUÇÃO DE BIODIESEL NA FABRICAÇÃO DE POLIURETANAS
}

\author{
A.A. MORANDIM-GIANNETTI ${ }^{1}$, A.M. PEDROZO ${ }^{1}$, L.B. $\operatorname{COSTA}^{1}$, L.S. SOARES ${ }^{1}$, L.P. \\ VITUSSO $^{1}$ \\ ${ }^{1}$ Centro Universitário do FEI - Departamento de Engenharia Química \\ E-mail para contato: preamorandim@fei.edu.br
}

\begin{abstract}
RESUMO - Com a procura por substituintes para os combustíveis atuais, a produção de biodiesel vem aumentando significativamente no país bem como a quantidade de glicerina. Assim, este trabalho visou à aplicação da glicerina no processo de fabricação de espumas flexíveis de poliuretana e a determinação de sua influência nas propriedades mecânicas. Dessa forma, após a obtenção da glicerina proveniente do processo de produção de biodiesel, a mesma foi separada e submetida a um processo de purificação parcial sendo a concentração determinada via HPLC antes da aplicação na produção das espumas. Após a obtenção das espumas foram realizados os ensaios mecânicos sendo observada a diminuição da densidade das espumas com a adição da glicerina e um aumento da força de indentação. Não foi observada diferença significativa da resiliência das espumas quando adicionada a glicerina. Sendo assim, as espumas confeccionadas com a utilização da glicerina residual do biodiesel foram consideradas comercialmente viáveis, pois suas propriedades não foram prejudicadas.
\end{abstract}

\section{INTRODUÇÃO}

O crescente consumo de biodiesel no país se dá devido à possível substituição do diesel de origem fóssil pelo biodiesel, reduzindo assim a emissão de gases de exaustão. Porém, o processo de produção do biodiesel tem como coproduto a "glicerina loira" (que compreende cerca de $80 \%$ de glicerol, além de álcool, água e outros sais inorgânicos do catalisador), que é produzida em grande quantidade. Esse fato acaba se tornando um obstáculo, pois para grande parte das aplicações, a glicerina precisa ser purificada (MOTA; GOLÇALVES; SILVA, 2009).

Dessa forma, para que a glicerina possa ser utilizada torna-se necessária sua purificação, procedimento que irá conferir um maior valor agregado a esse coproduto sendo que, a sequência de tratamentos mais utilizados em escala industrial é a neutralização da glicerina com ácidos (hidrólise ácida), seguido de evaporação ou destilação a vácuo, sendo a última a que confere uma pureza ao produto de aproximadamente 99,5\% (MENDES; SERRA, 2012).

Neste contexto, buscam-se aplicações em que a mesma não necessite de um elevado grau de pureza sendo, a produção de espumas flexíveis de poliuretana, uma possível fonte para o consumo da mesma, uma vez que, durante sua produção, uma diversificada combinação de 
monômeros, geralmente advindos da reação de um isocianato com um poliol, em associação com aditivos, são utilizados gerando diversos tipos de materiais (HERRIGTON; HOCK, 1997).

Sendo assim, a utilização da glicerina na produção de espumas flexíveis de poliuretana mostra-se como uma promissora aplicação, de forma que possa, em algum momento, vir a ter total absorção pelo mercado interno.

Diante dessa oportunidade, o presente trabalho que teve como objetivo o reaproveitamento da glicerina resultante da produção do biodiesel para o processo de fabricação de espumas flexíveis de poliuretana vem de encontro com as pesquisas realizadas atualmente visando o reaproveitamento de subprodutos de processos.

\section{MATERIAIS E MÉTODOS}

Inicialmente, empregando-se um reator de aço inox com capacidade máxima de 5L foi obtido o biodiesel utilizando-se uma proporção molar óleo/metanol de 1:6. A temperatura de reação foi de $50^{\circ} \mathrm{C}$ e o tempo de 30 minutos. O catalisador empregado foi o hidróxido de potássio $(\mathrm{KOH})$, numa fração mássica de $1 \%$ em relação à massa do óleo. Após o término da reação, o biodiesel e a glicerina foram separados, sendo a glicerina submetida ao processo de prépurificação via destilação simples, seguida de uma hidrólise ácida, na qual foi adicionada água destilada a $60^{\circ} \mathrm{C}$ e ácido fosfórico $\left(\mathrm{H}_{3} \mathrm{PO}_{4}\right) 85 \%$ e separadas as fases, seguida de outra destilação simples, com o objetivo de remover a água em excesso que foi adicionada anteriormente, sendo o resultado da destilação a glicerina pré-purificada.

Após esse processo, a glicerina proveniente de cada uma das etapas foi analisada via HPLC e via Espectrometria na Região do Infravermelho e utilizada para a produção das espumas flexíveis de poliuretana, utilizando-se, para isso, uma quantidade padrão de glicerina previamente determinada através de testes, a qual foi repetida em cada um dos experimentos identificados abaixo além do isocianato MDI (Specflex NE 134) e poliol poliéter (Specflex NF 964).

grupo 1 - Espumas com glicerina destilada;

grupo 2 - Espumas com glicerina pré-purificada;

grupo 3 - produção de espumas com a glicerina pura P.A.;

grupo 4 - espuma convencional, sem a adição de qualquer tipo de glicerina.

As espumas foram confeccionadas em triplicata, sendo obtido um total de 12 espumas, divididas em seus respectivos grupos. Para melhorar o modelo e confirmar os resultados dos testes foi feita mais uma espuma para o grupo 4, que corresponde ao grupo das espumas convencionais, sem a adição de glicerina, sendo todas as amostras submetidas a ensaios químicos e mecânicos utilizando-se uma série de testes padronizados segundo a Associação Brasileira de Normas Técnicas - ABNT (2003) (Tabela 1) 
Tabela 1: Formulação das espumas analisadas

\begin{tabular}{c|cccc|ccc|ccc|ccc}
\hline \multicolumn{10}{c}{ Partes } \\
\hline & \multicolumn{1}{c|}{ Convencional } & \multicolumn{1}{c}{ Glicerina P.A } & \multicolumn{3}{c}{ Glicerina Pré- } & \multicolumn{3}{c}{ Glicerina } \\
\hline Espuma & $\mathbf{1}$ & $\mathbf{5}$ & $\mathbf{9}$ & $\mathbf{1 3}$ & $\mathbf{2}$ & $\mathbf{6}$ & $\mathbf{1 0}$ & $\mathbf{3}$ & $\mathbf{7}$ & $\mathbf{1 1}$ & $\mathbf{4}$ & $\mathbf{8}$ & $\mathbf{1 2}$ \\
\hline Poliol & 100 & 100 & 100 & 100 & 98 & 98 & 98 & 98 & 98 & 98 & 98 & 98 & 98 \\
Isocianato & 35 & 35 & 35 & 35 & 50 & 50 & 50 & 50 & 50 & 50 & 50 & 50 & 50 \\
Glicerina & - & - & - & - & 2 & 2 & 2 & - & - & - & - & - & - \\
Glicerina & - & - & - & - & - & - & - & 2 & 2 & 2 & - & - & - \\
Glicerina & - & - & - & - & - & - & - & - & & - & 2 & 2 & 2 \\
\hline & \multicolumn{10}{c}{ Massa (g) } \\
\hline Poliol & 1600 & 1600 & 1600 & 1600 & 1411 & 1411 & 1411 & 1411 & 1411 & 1411 & 1411 & 1411 & 1411 \\
Isocianato & 560 & 560 & 560 & 560 & 720 & 720 & 720 & 720 & 720 & 720 & 720 & 720 & 720 \\
Glicerina & 0 & 0 & 0 & 0 & 29 & 29 & 29 & 0 & 0 & 0 & 0 & 0 & 0 \\
Glicerina & 0 & 0 & 0 & 0 & 0 & 0 & 0 & 29 & 29 & 29 & 0 & 0 & 0 \\
Glicerina & 0 & 0 & 0 & 0 & 0 & 0 & 0 & 0 & 0 & 0 & 29 & 29 & 29 \\
\hline
\end{tabular}

Dessa forma, as espumas obtidas foram caracterizadas via determinação da densidade segundo a norma ABNT - NBR 8537:2003, determinação da resiliência segundo a norma NBR 8619:2003 e determinação da Força de Indentação segundo a norma NBR 9176:2003.

\section{RESULTADOS E DISCUSSÕES}

Após a obtenção do biodiesel e separação das fases, foi realizada, inicialmente, a purificação da glicerina sendo verificado que à medida que a pureza da glicerina aumentava, era observado um clareamento da fase (Tabela 2).

Tabela 2: Porcentagem em massa das amostras de glicerina pelo método via HPLC

\begin{tabular}{lccc}
\hline \multicolumn{1}{c}{ Amostra } & Área de Pico & Massa (g) & \%Glicerina \\
\hline Glicerinassa) \\
& 50819562 & 77,15 & 64,08 \\
& 51983072 & 78,90 & 65,53 \\
Média & 50755910 & 77,06 & 64,00 \\
\hline Glicerina destilada & & & $\mathbf{6 4 , 5 4} \pm \mathbf{0 , 6 6}$ \\
& 69819140 & 105,65 & 76,34 \\
& 69282558 & 104,85 & 75,76 \\
Média & 69375150 & 104,99 & 75,86 \\
\hline Glicerina pré-purificada & 81232144 & 204,62 & 87,15 \\
& 113429910 & 228,09 & 89,45 \\
& 82282319 & 207,25 & 88,26 \\
Média & & & $\mathbf{8 8 , 2 9} \pm \mathbf{0 , 7 7}$ \\
\hline
\end{tabular}




\section{9 a 22 de outubro de 2014 \\ Florianópolis/SC}

Assim, após o processo de purificação e determinação da porcentagem de pureza das amostras de glicerina, foram obtidas as espumas de poliuretana sendo monitorado o tempo de crescimento e a altura obtida em cada caso (Tabela 3) sendo observado que as espumas produzidas com a glicerina destilada (espumas 4, 8 e 12) e pré-purificada (espumas 3, 7 e 11) apresentaram um menor tempo de crescimento, fato acarretado pela presença de traços de ácido graxo nessas glicerinas, que quando adicionadas a espuma fazem com que a amina contida na formulação seja neutralizada, produzindo o sal correspondente que atua como melhor catalisador da reação de expansão, acelerando assim a produção de gás carbônico e o crescimento da espuma.

Tabela 3: Tempo de crescimento e alturas das espumas

\begin{tabular}{cccccccccccccc}
\hline Espuma & $\mathbf{1}$ & $\mathbf{2}$ & $\mathbf{3}$ & $\mathbf{4}$ & $\mathbf{5}$ & $\mathbf{6}$ & $\mathbf{7}$ & $\mathbf{8}$ & $\mathbf{9}$ & $\mathbf{1 0}$ & $\mathbf{1 1}$ & $\mathbf{1 2}$ & $\mathbf{1 3}$ \\
\hline Tempo de & & & & & & & & & & & & & \\
Crescimento (min) & 1,21 & 2,18 & 2,01 & 1 & 1,33 & 2 & 1,54 & 1,09 & 2 & 1,55 & 1,4 & 1 & 1,97 \\
Altura (cm) & 25,4 & 29 & 32 & 30 & 26,2 & 29,5 & 32,5 & 32 & 26,5 & 28,7 & 33,5 & 32 & 26 \\
\hline
\end{tabular}

Para a determinação da influência da adição de glicerina em espumas convencionais foram analisadas a densidade, a força de indentação e a resiliência das amostras numeradas de 1 a 13, sendo os resultados analisados no programa estatístico JMP ${ }^{\circledR}$ PRO 10.0.2, pelo método All Pairs Turkey-Kramer. Desse método foram analisados três valores, o $\mathrm{R}^{2}$, o F Ratio e a Prob>F sendo que, para a realização dos mesmos, os corpos de prova foram cortados segundo as normas.

Assim, durante a determinação da densidade da espuma utilizou-se amostras de dimensões $10 \mathrm{~cm}$ x $10 \mathrm{~cm}$ x $5 \mathrm{~cm}$, sendo o volume de $500 \mathrm{~cm}^{3}$ e o peso de cada uma correspondente a média de três pesagens consecutivas, demonstrados na Tabela 4.

Tabela 4: Medidas das massas das espumas

\begin{tabular}{cccccccccccccc}
\hline Espuma & $\mathbf{1}$ & $\mathbf{2}$ & $\mathbf{3}$ & $\mathbf{4}$ & $\mathbf{5}$ & $\mathbf{6}$ & $\mathbf{7}$ & $\mathbf{8}$ & $\mathbf{9}$ & $\mathbf{1 0}$ & $\mathbf{1 1}$ & $\mathbf{1 2}$ & $\mathbf{1 3}$ \\
\hline $\begin{array}{c}\text { Medida } \\
\text { 1 (g) }\end{array}$ & 19,82 & 18,78 & 17,09 & 18,56 & 19,73 & 19,57 & 17,03 & 18,39 & 19,96 & 18,87 & 17,1 & 18,52 & 19,49 \\
$\begin{array}{c}\text { Medida } \\
\text { 2 (g) }\end{array}$ & 19,8 & 19,31 & 17,36 & 18,4 & 20,39 & 19,29 & 17,39 & 18,52 & 20,3 & 19,52 & 17 & 18,3 & 19,42 \\
Medida & & & & & & & & & & & & & \\
$\quad 3(\mathrm{~g})$ & 19,07 & 19,03 & 17,21 & 18,4 & 20,16 & 19,02 & 17,23 & 18,78 & 19,67 & 19,4 & 16,9 & 18,56 & 18,81 \\
Final (g) & 19,8 & 19,03 & 17,2 & 18,4 & 20,2 & 19,29 & 17,23 & 18,52 & 19,96 & 19,4 & 17 & 18,52 & 19,42 \\
\hline
\end{tabular}

Analisando-se os resultados pode-se observar uma diminuição no valor da massa das espumas com glicerina em relação à espuma convencional. Tal fato pode ser explicado pela presença da glicerina tornar a formulação mais reativa, devido a maior presença de hidroxilas livres, alterando assim a viscosidade da mistura, o que faz com que essa atinja a gelificação mais rapidamente, ficando mais massa no recipiente de metal antes de despejar o conteúdo na caixa, ocasionando perdas de material. Dessa forma, após a obtenção dos resultados, foi calculada a 
densidade aparente em cada caso (Tabela 5) e, através da análise estatística, pode-se afirmar que as espumas são estatisticamente diferentes quanto à densidade, utilizando-se o método TurkeyKramer (Figura 1).

Tabela 5: Densidade aparente das amostras de espuma

\begin{tabular}{cccccccccccccc}
\hline Espuma & $\mathbf{1}$ & $\mathbf{2}$ & $\mathbf{3}$ & $\mathbf{4}$ & $\mathbf{5}$ & $\mathbf{6}$ & $\mathbf{7}$ & $\mathbf{8}$ & $\mathbf{9}$ & $\mathbf{1 0}$ & $\mathbf{1 1}$ & $\mathbf{1 2}$ & $\mathbf{1 3}$ \\
\hline Densidade $\left(\mathrm{Kg} / \mathrm{m}^{3}\right)$ & 39,6 & 38,06 & 34,42 & 36,8 & 40,32 & 38,58 & 34,46 & 37,04 & 39,92 & 38,8 & 34 & 37,04 & 38,84 \\
\hline
\end{tabular}

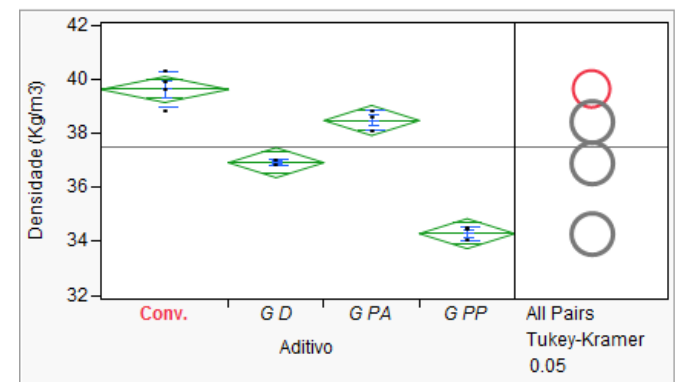

Figura 1: Análise da densidade das amostras

Observa-se também uma visível diminuição na densidade das espumas com glicerina, fato que ocorre devido à presença de ácidos graxos que neutralizam a amina, produzindo um sal que atua como um catalisador mais eficiente que a amina na reação entre o isocianato e a água, o que leva a formação de maior quantidade de ácido carbâmico no processo de expansão, que se decompõe em gás carbônico, o que consequentemente diminui sua densidade sendo importante ressaltar que o valor de $\mathrm{R}^{2}$, F Ratio e de Prob $>$ F obtidos no programa estatístico revela que os dados de densidade podem ser explicados pelo método utilizado, já que $\mathrm{R}^{2}$ é bem próximo de $1 \mathrm{e}$ prob> F pequeno o suficiente para ser desprezível, devido ao fato de grande parte da variância poder ser explicada pelo modelo. Esses valores podem ser vistos na Tabela 6.

Tabela 6: Resultados da análise da densidade

\begin{tabular}{cc}
\hline \multicolumn{2}{c}{ Análise } \\
\hline $\mathrm{R}^{2}$ & 0,97 \\
F Ratio & 97,45 \\
Prob $>$ F & $<0,001$ \\
\hline
\end{tabular}

Outro teste realizado foi o de determinação da resiliência das espumas confeccionadas, sendo utilizados corpos de provas com dimensões de $10 \mathrm{~cm}$ x $10 \mathrm{~cm}$ x $5 \mathrm{~cm}$, conforme a norma NBR 8619:2003 (Tabela 7) sendo que, a partir da análise pelo método Turkey-Kramer (Figura 2), conclui-se que a quantidade de glicerina adicionada às espumas não foi suficiente para provocar uma alteração nessa propriedade. Dessa forma, pode-se observar na Tabela 8 que os valores de $\mathrm{R}^{2}$, F Ratio e de Prob>F não estão dentro do considerado aceitável, pois o modelo não consegue explicar os resultados quando não há uma diferença significativa entre eles. 
Tabela 7: Resiliências das espumas

\begin{tabular}{crrrrrrrrrrrrr}
\hline Espuma & $\mathbf{1}$ & $\mathbf{2}$ & $\mathbf{3}$ & $\mathbf{4}$ & $\mathbf{5}$ & $\mathbf{6}$ & $\mathbf{7}$ & $\mathbf{8}$ & $\mathbf{9}$ & $\mathbf{1 0}$ & $\mathbf{1 1}$ & $\mathbf{1 2}$ & $\mathbf{1 3}$ \\
\hline Medida 1 (\%) & 45 & 43 & 45 & 45 & 53 & 48 & 50 & 55 & 41 & 55 & 51 & 55 & 46 \\
Medida 2(\%) & 44 & 51 & 43 & 43 & 50 & 43 & 46 & 58 & 44 & 59 & 53 & 50 & 50 \\
Medida 3 (\%) & 44 & 44 & 43 & 44 & 50 & 54 & 43 & 54 & 48 & 58 & 49 & 52 & 49 \\
Final (\%) & 44 & 44 & 43 & 44 & 50 & 48 & 46 & 55 & 44 & 58 & 51 & 52 & 49 \\
\hline
\end{tabular}

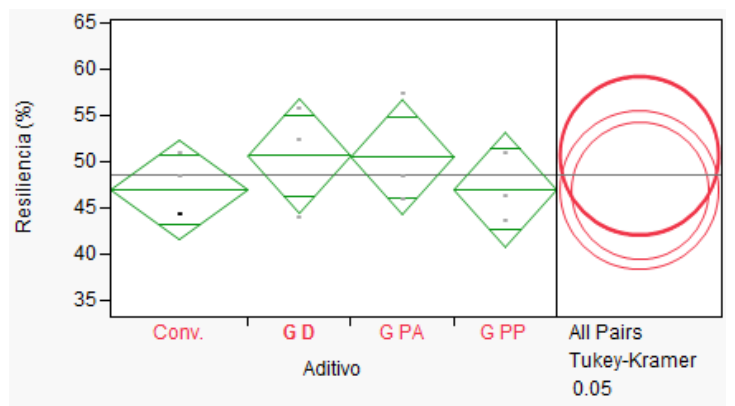

Figura 2: Análise da resiliência

Tabela 8: Resultados da análise da resiliência

\begin{tabular}{cc}
\hline \multicolumn{2}{c}{ Análise } \\
\hline $\mathrm{R}^{2}$ & 0,171 \\
F Ratio & 0,622 \\
Prob $>$ F & 0,6185 \\
\hline
\end{tabular}

Além do teste de densidade e de resiliência, foi determinada a força de indentação utilizando corpos de prova de dimensões $380 \mathrm{~mm}$ x $380 \mathrm{~mm}$ x $50 \mathrm{~mm}$ submetidos a ensaios no equipamento Instron 5565, determinando assim valor de força $(\mathrm{N})$ que quando aplicada cause $25 \%, 40 \%$ ou $65 \%$ de deformação sobre a altura inicial. Esses valores estão demonstrados na Tabela 9.

Tabela 9: Análise da força de indentação

\begin{tabular}{cccccccccccccc}
\hline Espuma & $\mathbf{1}$ & $\mathbf{2}$ & $\mathbf{3}$ & $\mathbf{4}$ & $\mathbf{5}$ & $\mathbf{6}$ & $\mathbf{7}$ & $\mathbf{8}$ & $\mathbf{9}$ & $\mathbf{1 0}$ & $\mathbf{1 1}$ & $\mathbf{1 2}$ & $\mathbf{1 3}$ \\
\hline FID 25\% (N) & 21 & 42 & 31 & 27 & 17 & 41 & 30 & 29 & 16 & 41 & 28 & 24 & 12 \\
FID 40\% (N) & 32 & -64 & -46 & -42 & -27 & -62 & -46 & -46 & -26 & -61 & -43 & -37 & -21 \\
FID 65\% (N) & -82 & -162 & -118 & -107 & -73 & -158 & -119 & -118 & -70 & -157 & -111 & -95 & -62 \\
\hline
\end{tabular}

Os valores referentes à deformação de $40 \%$ foram analisados no programa estatístico JMP, muito utilizado comercialmente para efeito de comparação. Os resultados são demonstrados na Figura 3. 


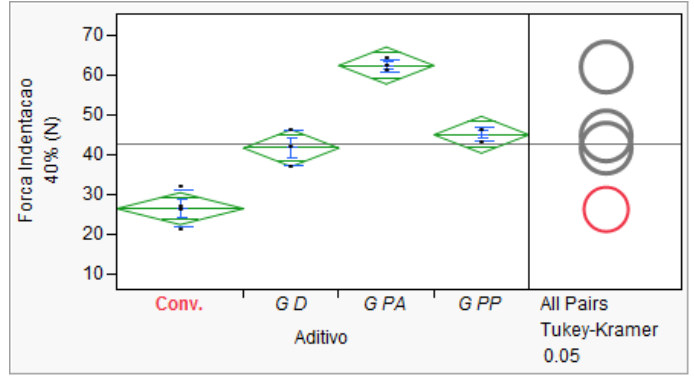

Figura 3: Análise da força de indentação.

Analisando-se os resultados obtidos, pode-se observar que as espumas com glicerina apresentam uma maior força de indentação para deformar em $40 \%$, ou seja, sua presença faz com que ocorram ligações cruzadas. Além disso, por ter uma cadeia curta, a glicerina acaba gerando ramificações, aumentando as ligações na espuma, proporcionando um fechamento que a torna mais resistente, precisando assim de uma maior força para deformá-la. Também é possível observar uma consistência nos resultados, uma vez que as espumas com glicerina apresentam uma resistência crescente de acordo com o aumento da quantidade de glicerina, levando-se em consideração que quanto maior a pureza, maior a sua quantidade na espuma.

Quando analisados os valores de $\mathrm{R}^{2}$, F Ratio e Prob>F, pode-se dizer que os dados podem ser explicados pelo método adotado, já que $\mathrm{R}^{2}$ está próximo de 1 , assim como a maior parte da variância pode ser explicada pelo modelo e Prob>F é pequeno o suficiente para ser desprezível. Esses valores podem ser vistos na Tabela 10.

Tabela 0: Resultados da análise da força de indentação

\section{CONCLUSÕES}

\begin{tabular}{cc}
\hline \multicolumn{2}{c}{ Análise } \\
\hline $\mathrm{R}^{2}$ & 0,951 \\
F Ratio & 59,419 \\
Prob $>$ F & $<0,001$ \\
\hline
\end{tabular}

Ao comparar-se com a glicerina obtida diretamente do biodiesel (loira), antes de realizarse a destilação simples; e depois de realizada a destilação (glicerina destilada), essas glicerinas apresentaram pureza inferior como esperado sendo que a presença dessa impureza afetou nas propriedades das espumas, uma vez que interfere na ação da amina, que é o catalisador da reação entre o isocianato e a água.

Quando realizados os testes mecânicos, foi observada uma diminuição na densidade das espumas com glicerina, devido à presença dos ácidos graxos que interferem na reação, formando maior quantidade de gás no processo de expansão da espuma. Já no teste de resiliência, a quantidade de glicerina adicionada não foi suficiente para provocar alterações significativas nessa propriedade. No teste de força de indentação, observou-se que a força necessária para deformar 
em $40 \%$ a espuma aumentou quando houve o aumento da quantidade de glicerina na espuma, fato que pode ser explicado pela presença de maior quantidade de ligações cruzadas na espuma.

Dessa forma, os testes mostraram que as espumas confeccionadas com as amostras de glicerina provenientes da produção do biodiesel podem ser utilizadas comercialmente, pois as propriedades alteradas não prejudicam a sua aplicação, apenas modificam a sua funcionalidade, como, por exemplo, um colchão mais macio ou um colchão mais resistente, mais duro. Contudo, alguns fatos devem ser estudados e observados com atenção, como a quantidade de glicerina a ser adicionada, pois como já citado, ela pode causar perda do material, queima do centro da espuma e risco de pegar fogo, colocando em risco o processo e as instalações.

Assim, a aplicação de glicerina residual para a produção de espumas flexíveis de poliuretana se faz possível e é comercialmente viável, uma vez que não apresentou mudanças nas propriedades que prejudicassem a qualidade e a utilização do material.

\section{REFERÊNCIAS}

ASSOCIAÇÃO BRASILEIRA DE NORMAS TÉCNICAS. NBR 8537: espuma flexível de poliuretano - determinação da densidade. Rio de Janeiro, jul. 2003. jul. 2003.

.NBR 8619: espuma flexível de poliuretano - determinação da resiliência. Rio de Janeiro,

.NBR 9176: espuma flexível de poliuretano - determinação da força de indentação. Rio de Janeiro, jul. 2003.

.NBR 9177: espuma flexível de poliuretano - determinação da fadiga dinâmica. Rio de Janeiro, jul. 2003.

HERRINGTON, Ron; HOCK, Kathy. Flexible Polyurethane Foams. $2^{\mathrm{a}} \mathrm{ed}$. Texas: The Dow Chemical Company, 1997.

MENDES, Danylo; SERRA, Juan. Glicerina: uma abordagem sobre a produção e o tratamento. Revista Liberato, Novo Hamburgo, v. 13, n. 20, jul./dez. 2012. Disponível em: <http://www.liberato.com.br/upload/arquivos/0107121220302827.pdf>. Acesso em: 11 mar. 2013.

MOTA, Claudio; GOLÇALVES, Valter; SILVA, Carolina. Gliceroquímica: novos produtos e processos a partir da glicerina de produção de biodiesel. Química Nova, v. 32, n. 3, p. 639-648, mar. 2009. Disponível em: <http://www.scielo.br/pdf/qn/v32n3/a08v32n3.pdf>. Acesso em: 05 mar. 2013. 